\title{
Blepharoplasty incorporating epicanthoplasty using the scar-hiding procedure
}

Background: Epicanthoplasty incorporating blepharoplasty is the most popular option of cosmetic surgeries in Asians. Thus, it is very important to develop a scar-hiding procedure. Here, we introduce a new procedure for correcting epicanthus folds incorporating blepharoplasty to hide post-surgical scars.

Method: We retrospectively compared post-surgical scars on the epicanthus, using the Stony Brook Scar Evaluation Scale (SBSES), between this procedure and the Y-V procedure. A total of 342 patients underwent blepharoplasty incorporating epicanthoplasty using this scar-hiding procedure from September 2013 to December 2014 in our department (group A). And 260 patients' follow-up period is longer than 6 months. A total of 278 patients underwent Y-V epicanthoplasty incorporating blepharoplasty from September 2011 to October 2012 (group B). And 189 patients' follow-up period is longer than 6 months. On the 3rd month and 6th month after surgery, patients indicated their satisfaction with the scar on the canthus area by the standard $100 \mathrm{~mm}$ visual analog scale (VAS). In addition, the scar on the medial canthus area was evaluated by two anonymous, independent plastic surgeons based on digital images by using Stony Brook Scar Evaluation Scale (SBSES). These surgeons were not part of the study.

Results: Patients were significantly more satisfied with the scar-hiding procedure than the $\mathrm{Y}-\mathrm{V}$ procedure, as indicated by the VAS scores (95.2 vs. 90.5, respectively, $\mathrm{p}<0.05$ ). Moreover, the scar-hiding procedure achieved better SBSES scores.

Conclusions: The scar-hiding procedure is easy to design and perform, and completely hides post-surgical scars. It is recommended for Asian people with epicanthus. Level of Evidence: Level V, clinical experience.

\section{Keywords: epicanthoplasty, blepharoplasty, post-surgical scar}

\section{Introduction}

The epicanthus is a semilunar fold of skin across from the upper eyelid to the lower eyelid on the medial canthus area. It is often congenital and transmitted as a dominant trait. It usually occurs in people without superior palpebral fold, particularly in the Asians [1]. Epicanthoplasty incorporating blepharoplasty is the most popular option of cosmetic surgeries. And hypertrophic scars are more likely to occur in the Asians than in the Caucasians. Thus, it is very important to develop a scar-hiding procedure.

\section{Materials and methods}

A total of 342 patients underwent blepharoplasty incorporating epicanthoplasty using this scar-hiding procedure from September 2013 to December 2014 in our department (group A). And 260 patients' follow-up period is longer than 6 months. A total of 278 patients underwent $\mathrm{Y}-\mathrm{V}$ epicanthoplasty incorporating blepharoplasty from September 2011 to October 2012 (group B). And 189 patients' follow-up period is longer than 6 months.

\section{Surgical techniques}

The surgery was performed under local anesthesia and all patients were in the supine position. All sutures were removed on the 6th days after the surgery.

\section{- Group A: The scar-hiding procedure}

The incision marks were made with a fine felt-tip syringe marker. The patient should keep the eyes open or closed following requests of the surgeon during marking. The medial canthus fold was spread out by pulling the nasal skin super-medially. Point A was the medial-most point of the lacrimal lake (FIGURES 1A and 1E). Point $A^{\prime}$ was the surface representation of
Fang Yan Jia ${ }^{1}$, Lei Xiong ${ }^{2}$, Li Hong Qiu ${ }^{3}$, Jin Yan ${ }^{1}$, Jialiang $\mathrm{Chen}^{3}$ \& Cheng Gang Yi*3

${ }^{1}$ Department of Plastic and cosmetic surgery, Xi'an Jiao Tong University affiliated the second hospital, China

${ }^{2}$ Department of ophthalmology, Xi'an Jiao Tong University affiliated the second hospital, China

${ }^{3}$ Institute of Plastic Surgery Xi Jing Hospital, Fourth Military Medical University, China

*Author for correspondence: yichg@163.com 
Point A (FIGURES 1B and 1F). Line AA' was marked (FIGURES $1 \mathrm{~B}$ and 1H). Point $\mathrm{C}$ was about $1 \mathrm{~mm}$ under inferior punctum (FIGURES 1C and 1D). Line AC was a curve line paralleling to the lower lid margin (FIGURES 1G).

A

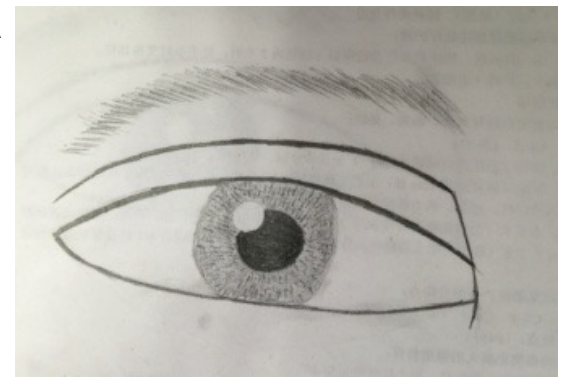

C

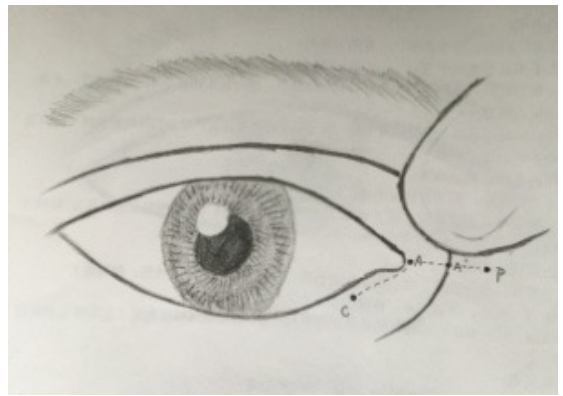

E

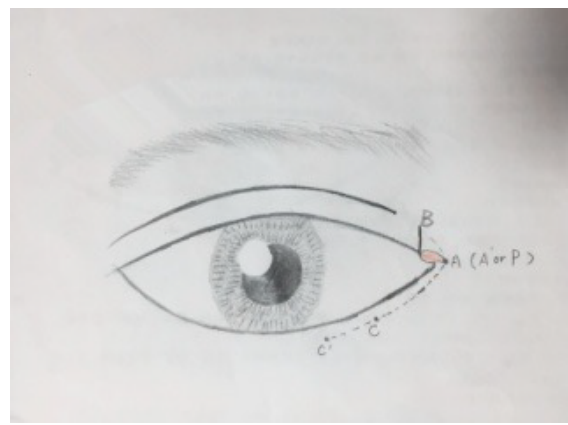

G

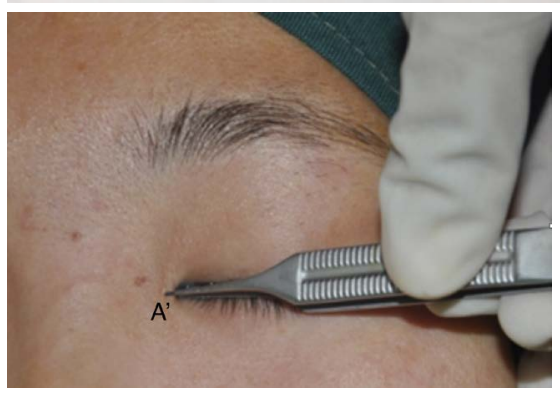

I

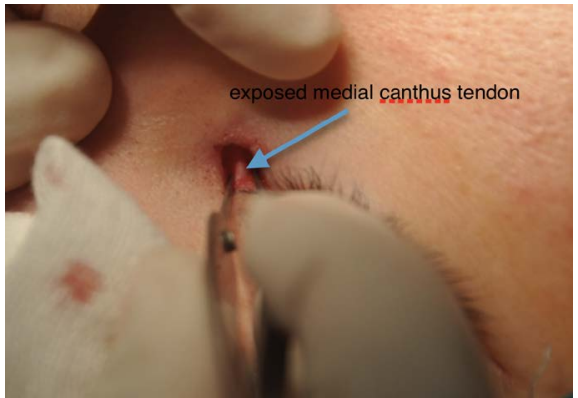

B

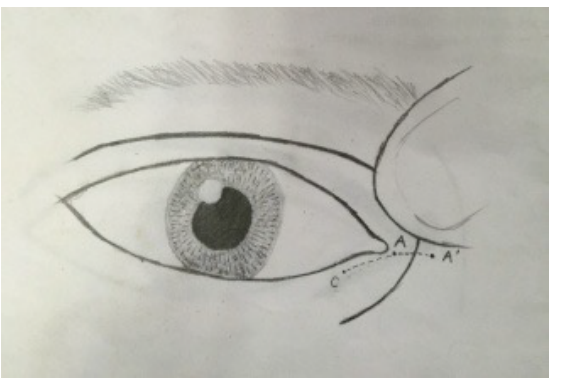

D

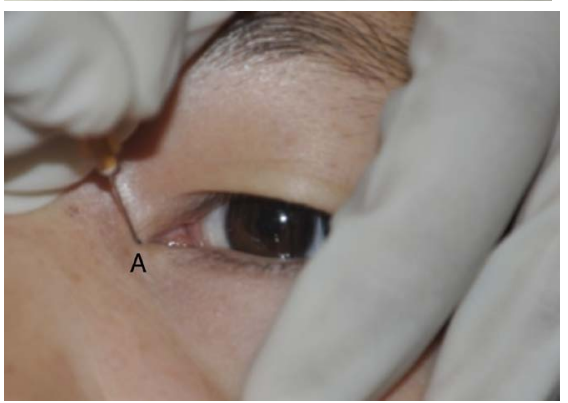

$\mathbf{F}$

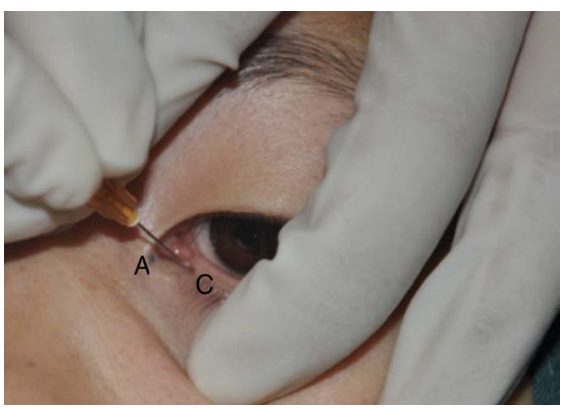

H

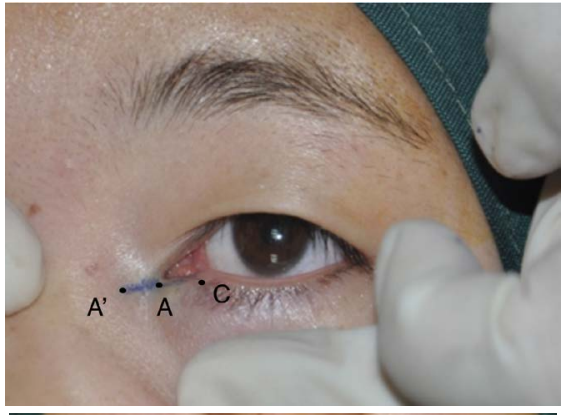

J

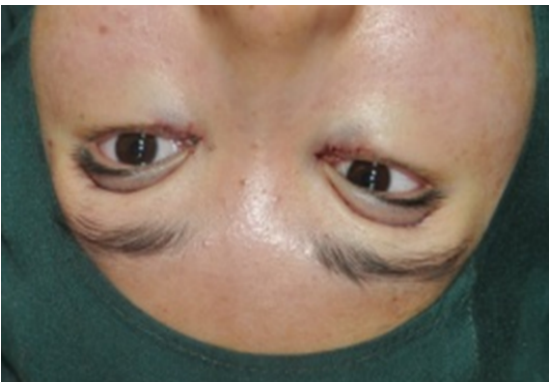

FIGURE 1A-J. Group A: The scar-hiding procedure. 
scissors. Dislocated orbicularis oculi muscle was cut off, so the tension crossing the epicanthus was released completely and the superficial medial canthus tendon. Then the medial canthus skin was repositioned spontaneously to some extent.

In most cases, Point $\mathrm{A}$ was covered by Point $A^{\prime}$ after the dissection. If the lacrimal lake is not exposed enough, the medial canthus tendon would be folded. Then Point $P$ was marked as the new medial canthus present point depending on the surgeon's experience. The incision line A'P was made and point $A$ and Point $P$ were placed together without tension. But if the lacrimal lake was exposed enough, the medial canthus tendon should not to be folded.

Sometimes, Point A wasn't covered by Point $A^{\prime}$, Point $A^{\prime}$ was viewed as the medial canthus present point. Then, the medial canthus tendon was plicate in moderation. Point $A$ and Point $A^{\prime}$ were placed together without tension.

The lower lid incision was extended to the lateral and the tissue below the incision Line CC' was raised up between the skin and muscle. The skin flap was stretched and excess skin was removed off when the patient was asked to gaze straight ahead. The finished point was Point C' (FIGURE 1D and 1I). A clear 7-0 permanent nylon suture was placed.

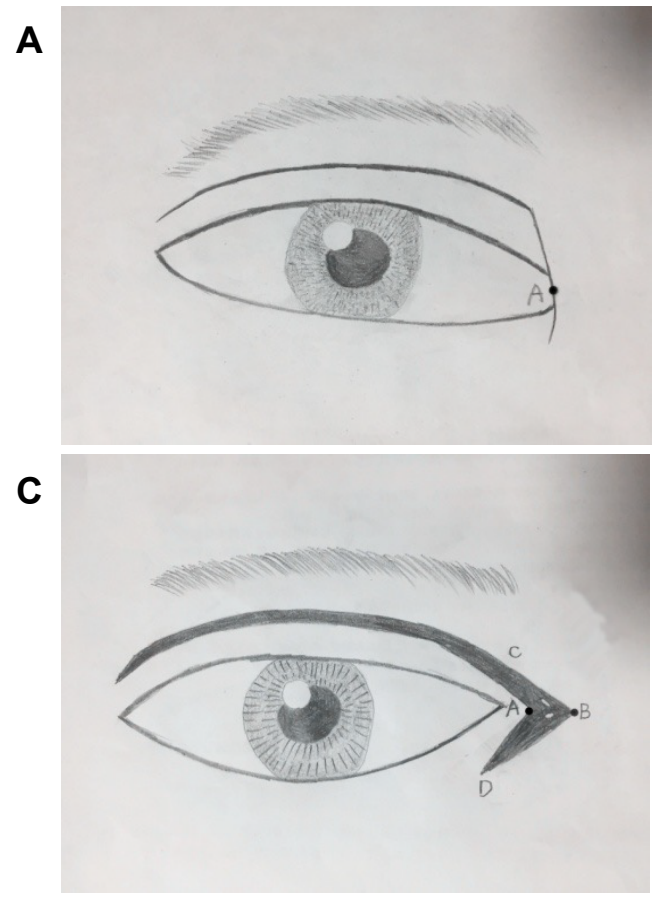

FIGURE 2A-D. Group B: Y-V advanced procedure.
After the blepharoplasty, the dog-ear on the upper lid was removed directly to finish at point $B$ (FIGURE 1D and 1J). The curvilinear Line $A B$ was consistent with the supra-tarsal fold, but the incisions were not linked. A clear 7-0 permanent nylon suture was placed.

\section{Group B: Y-V advanced procedure}

The jointed point of the Y-V plasty (Point A) was marked on the epicanthus margin at the level of the mid-caruncle (FIGURE 2A). Point B was marked as the new medial canthus position, which was located individually (FIGURE 2B). Line $\mathrm{AC}$ and Line $\mathrm{AD}$ were marked paralleling the upper palpebral margin and lower palpebral margin respectively. Line AC corresponded with the planned double eyelid fold (FIGURE 2C and 2D).

The incision was made through the skin. The dense fiber between the skin and muscle was dissected, so that the skin on the medial canthus area was raised up [2]. Tension crossing the epicanthus was released completely. Excess orbicularis oculi muscle was cut off, and fibrofatty tissue was subsequently released. The subcutaneous tissue of Point A was sutured to medial canthus tendon, which make sure the lacrimal lake exposed. If Point $A$ was covered by point $\mathrm{B}$, Line $\mathrm{AB}$ can be extended medially.

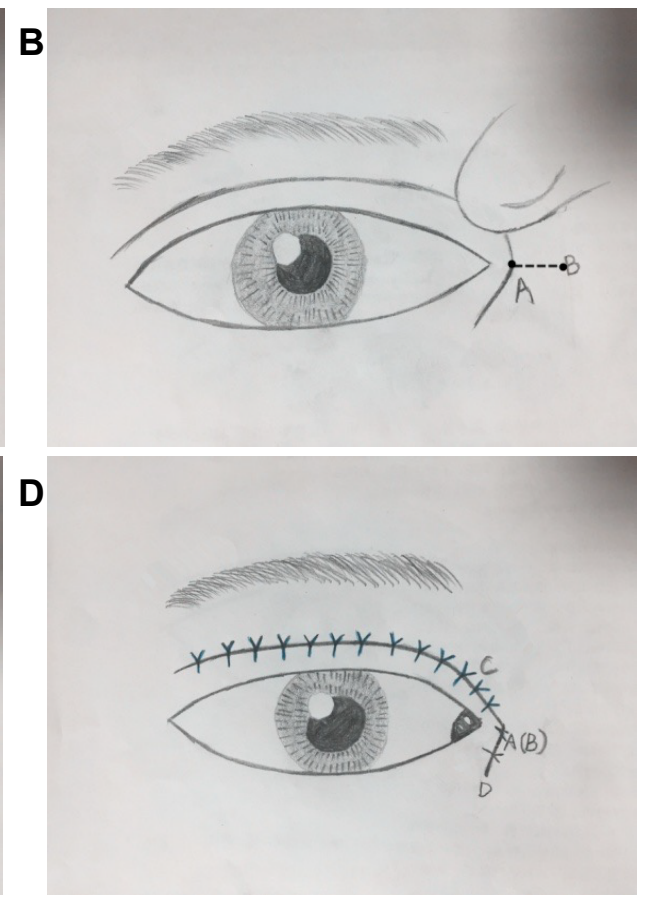


Then, Point A and Point B were sutured together without tension. The two triangular areas of skin were trimmed off. A clear 7-0 permanent nylon suture was placed.

\section{Results}

No recurrence of epicanthus and no disturbances in lacrimal function were observed. Most patients obtained satisfactory results.

On the 3rd month and the 6th month after surgery, patients indicated their satisfaction with the scar on the canthus area by the standard $100 \mathrm{~mm}$ visual analog scale (VAS)[3]. The left end of the $100 \mathrm{~mm}$ VAS scale means extremely dissatisfaction and the right end means full satisfaction. The scores were derived by measuring the distance from the left end of the scale.

In addition, the scar on the medial canthus area was evaluated by two anonymous, independent plastic surgeons. These surgeons were not part of the study. They looked at post- surgical images of the third month and the sixth month on a large screen. The cosmetic appearance scale was Stony Brook Scar Evaluation Scale (SBSES) [4], which was made up of five categories (TABLE 1). The total score was obtained by adding the scores ( 0 or 1$)$ of these five categories, ranging from 0 to 5 .

Patients were significantly more satisfied with the scar-hiding procedure than the Y-V procedure, as indicated by VAS score (95.2 vs. 90.5, respectively, p<0.05) (TABLE 2). Moreover, the scar-hiding procedure achieved better grade than the Y-V procedure, according to observers grading with the SBSES (TABLE 2).

The epicanthus is a semilunar fold of skin across from the upper eyelid to the lower eyelid on the medial canthus area. It is often congenital and transmitted as a dominant trait. It usually occurs in people without the supra-tarsal crease, particularly in Asians. The rates of epicanthus are about $50 \%$ in Asians and up to $70 \%$ in people with no supra-tarsal crease.

There are following aesthetic effects of epicanthus (FIGURE 3A-3E) [5]

1. Making the fissure short and the palpebral narrow and increasing the inter-canthus distance;

2. Making the medial eyelid obtuse and the
Table 1: Stony Brook Scar Evaluation Scale (SBSES).

\begin{tabular}{|l|c|}
\hline Scar Category & No. of Points \\
\hline Width & \\
\hline $2 \mathrm{~mm}$ & 0 \\
$\leq 2 \mathrm{~mm}$ & 1
\end{tabular}

$\leq 2 \mathrm{~mm} \quad 1$

Height

Elevated or depressed in relation to \begin{tabular}{l|l} 
surrounding skin & 0
\end{tabular}

\begin{tabular}{|l|l}
\hline Flat & 1
\end{tabular}

Color

Darker than surrounding skin (red,

purple, brown, or black)

Same color or lighter than surrounding

$\begin{array}{ll}\text { skin } & 1\end{array}$

Hatch marks or suture marks

\begin{tabular}{l|l}
\hline Present & 0 \\
\hline Absent
\end{tabular}

\begin{tabular}{|l|l}
\hline Absent & 1
\end{tabular}

Overall appearance

\begin{tabular}{l|l}
\hline Poor & 0
\end{tabular}

$\begin{array}{ll}\text { Good } & 1\end{array}$

Total score = sum of individual scores;

range, 0 (worst) to 5 (best)

\begin{tabular}{|c|c|c|c|}
\hline & Group A & Group B & $\mathbf{P}$ \\
\hline VAS & $95.2 \pm 14.2$ & $90.5 \pm 8.6$ & $<0.05$ \\
\hline SBSES (observer 1) & $4.8 \pm 1.2$ & $4.0 \pm 1.6$ & $<0.05$ \\
\hline SBSES (observer 2) & $4.9 \pm 1.1$ & $4.2 \pm 1.2$ & $<0.05$ \\
\hline
\end{tabular}

medial palpebral margin higher than the lateral, which makes people look sad;

3. Decreasing the corneal show rate, this makes people look tired;

4. Lesser exposure of the medial sclera than the lateral one, which makes people look "crossed-eyed"; and making the nasal bone look lower

A blepharoplasty without correcting epicanthus usually generates an impression of round palpebral fissure accompanied by shorter palpebral fissure and narrow supra-tarsal fold; thereby significantly affect the effects of surgery. Thus, epicanthoplasty incorporating blepharoplasty is the most popular option of cosmetic surgeries.

The origin of Asian epicanthus remains unclear. Yoo [6] suggested that the epicanthus represents a series of defects in development, including abnormally poor development of nasal 
A

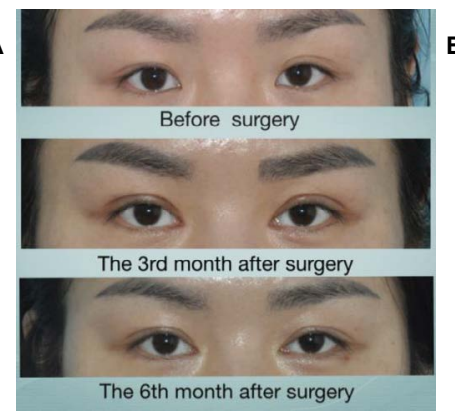

B
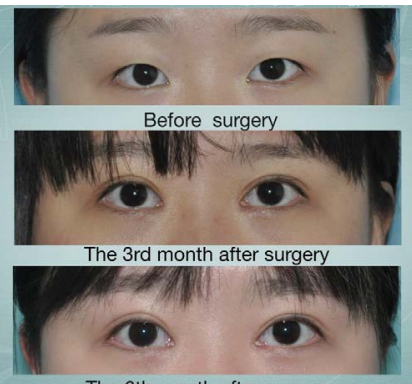

The 6th month after surgery

C

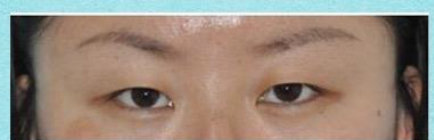

before surgery

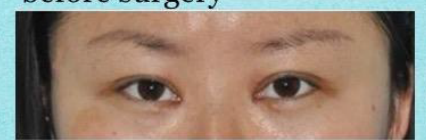

the 3 rd month post-surgery

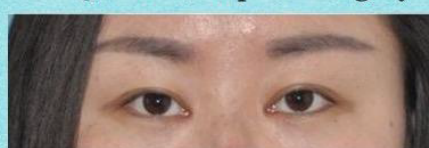

the 6 th month after surgery

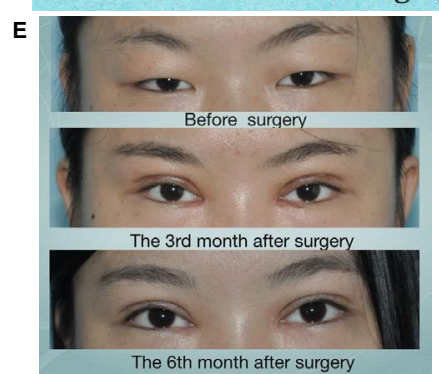

F

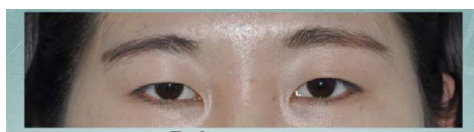

Before surgery

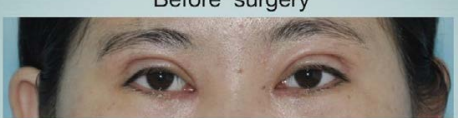

The 3rd month after surgery

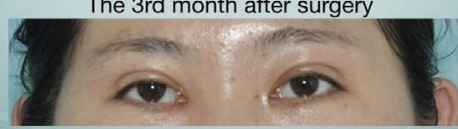

The 6th month after surgery
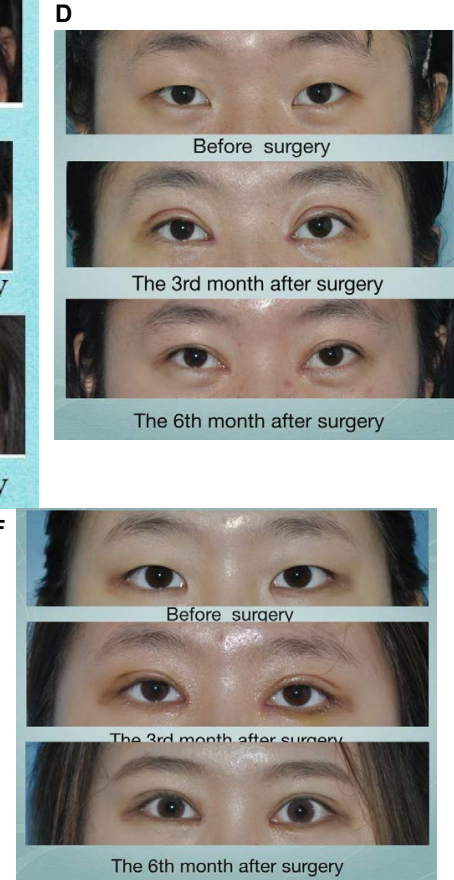

H

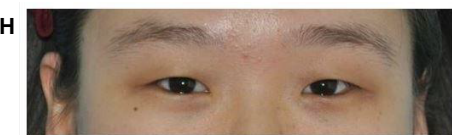

(2)

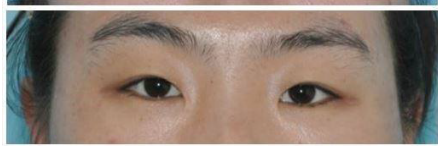

aesthetic effects of epicanthus
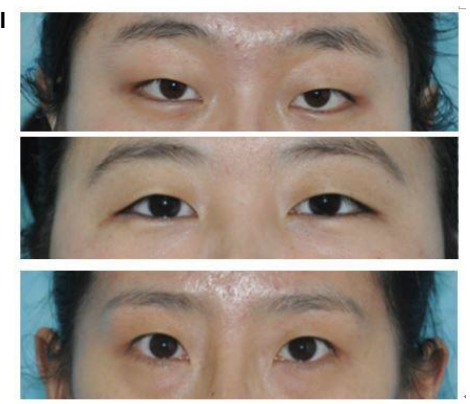

aesthetic effects of epicanthus

FIGURE 3-I. Aesthetic effects of epicanthus. 
bone, excess development of skin on the canthus area, attached dislocation of orbicularis oculi muscle and excess fibro-fatty tissue underlying the fold. Some Chinese anatomical study $[7,8]$ found that connective tissue and dense muscle fibers run obliquely over the medial canthus tendon from the upper eyelid to the lower eyelid, and they are located inside the epicanthus. So dislocation of the orbicularis oculi muscle at the insertion point of the canthus tendon may be the reason for epicanthus.

Several epicanthus corrective procedures have been introduced, such as modified Z-plasty method [9], Mustard's four flap method [10] and its modification[11-13], V-M plasty [14], Y-V advancement[15-18], and W-plasty[19]. These methods have complex incision designs and inevitably cause scars on the medial canthus area, which leads to unpleasant aesthetic appearance because of hypertrophic scar tendency of Asians. These procedures create the out-fold type of double eyelid fold which is not suited for most Asians. Thus, these procedures are no longer popular with Asians [20], and it is very important to develop an appropriate scarhiding procedure.

A Y-V procedure can be designed easily but the tension increases because of the excised skin, which increases the rate of hypertrophic scars; and the incisions are also visible. The scar contraction occurs frequently because of linked epicanthoplasty and blepharoplasty incisions, which makes the medial palpebral fissure look weird (FIGURE 3F).

Our patients were significantly more satisfied with the scar-hiding procedure than the $\mathrm{Y}-\mathrm{V}$ procedure, as indicated by the VAS scores. Moreover, the scar-hiding procedure achieved better SBSES scores.

The incision design for our scar-hiding procedure is very simple, and all incisions are hidden when the eyes are open. The supraciliary incision is consistent with the supra-tarsal fold and the sub-tarsal incision is near the sub eyelash, which is invisible.

Particular attention should be paid when dissecting the medial canthus area, to make sure the tension was released completely. We did not determine the final canthus position before performing the operation. Because the medial canthus area skin will reposition itself spontaneously to some extent after the attachment is released.

The dissection range near Line AA' should not be too large, or the scar contraction may lead to ectropion, which may make the incision visible and make lacrimal lake exposed too much that looks weird. The dissection should not be too deep to damage the sub lacrimal ductile. If the sub lacrimal ductile was fracture, the result would be disastrous. The patient cannot accept the consequence of epiphora after the epicanthoplasty and the repair of sub lacrimal ductile is very hard.

In most cases, Point $\mathrm{A}$ was covered by Point $A^{\prime}$ after the dissection. If the lacrimal lake is not exposed enough, the medial canthus tendon will be folded. Then Point $P$ is marked as the new medial canthus present point .It is not necessary to put Point $\mathrm{B}$ on the horizontal extension line of Line AA'. It depends on the surgeon's experience, which needs to consider the patient's aesthetic value and make sure point $A$ and Point $P$ are placed together without tension.

According the experience of Park [21] the new medial canthus present point needs to be confined to the thin skin. If the incision is extended onto the thick nasal skin, it is intend to create a hypertrophic scar.

The PDS suture was used to plicate the medial canthus tendon because it can maintain tension longer than other absorbable suture, thus reduce the risk of recurrence. And its histocompatibility is very good. It can avoid induration caused by the foreign body rejection. Its breakdown products are anti-bacterial, which prevents the hypertrophic scar.

With this procedure, we did not determine the final canthus position before performing the operation because the medial canthus area skin would reposition itself spontaneously to some extent after the attachment was released. The new medial canthus point was marked after completely dissection, so the appearance of medial canthus area could be controlled more precisely. It is easier to manage compared to other procedures which need to mark the final canthus point before surgery, especially for young surgeons.

The sub-tarsal incision is about $1 \mathrm{~mm}$ under the eyelash. If it is closer, the root of eyelash may be hurt, leading to eyelash direction disorder 
and even trichiasis. And the tissue is too hard for the anesthetic to be injected, which leaves the patient severe pain. If the incision is further to the eyelash, the incision will be visible.

When the excess skin under the lower lid incision was removed, the patient was required to gaze straight ahead. If the patient looked towards his head, the skin removed was less than the needed mount, and the excess skin would stack near the incision and form wrinkles. But if the patient looked towards his feet, the skin removed was less than the needed mount, which led to isolation of eyelid from eyeball and the consequence of epiphora. But for the patient suffered from the trichiasis of lower lid, the incision could be extended to the lateral canthus, and the skin removed could be more than normal to treat trichiasis.

Then, the upper lid dog-ear was removed directly to finish at Point B (FIGURE 3F-3I). The curvilinear line $\mathrm{AB}$ was consistent with the supra-tarsal fold, but the incisions were not linked. A clear 7-0 permanent nylon suture was placed. And the orbicularis oculi muscle cannot be removed too much to make the incision linked to the blepharoplasty incision under the skin. Thus the appearance on the medial canthus area wouldn't be affected by the post-surgical scar contraction (FIGURE 4). The reason why this step was made after blepharoplasty was to avoid its affection to the distribution of the skin on the medial canthus area. Thus the amount of
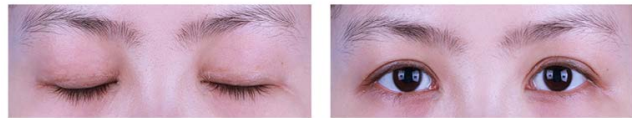

FIGURE 4.Y-Vplasty 2.5 years after surgery

tissue removed off could be more accurate and the result of the surgery would be better.

The medial canthus area was pressed by rolled Vaseline gauze after the surgery. The purpose is to prevent the tissue swollen, which could make the tissue sheared by the suture and then the appearance on medial canthus area destroyed.

According to our statistics, the patients were highly satisfied with the effect and the scar after the surgery. And the scores are much more satisfactory at the 6th month than that at the 3rd month after surgery.

This procedure is suitable for mild to moderate epicanthus. However, conventional procedures should be used to correct severe epicanthus deformities, such as epicanthus inversus and congenital anomalies.

Our scar-hiding procedure is simple to design and perform and completely avoids scars. This procedure is recommended for Asians with ethnic epicanthus.

\section{Acknowledgment}

The authors declare no conflicts of interest. 


\section{REFERENCES}

Xue T. A survey about epicanthus of 1731 primary and middle school students Chin. J. Plast. Surg. Burns. 4, 318-31' (1999).

Li FC, Ma LH. Double eyeli blepharoplasty incorporatin epicanthoplasty using Y-V advancemen procedure. J. Plast. Reconstr. Aesthet. Surદ 61(8), 901-905 (2008).

Faiz KW. [VAS--visual analog scale] Tidsskr Nor Laegeforen 134(3), 323 (2014)

Singer AJ, Arora B, Dagum A, et a Development and validation of a nov scar evaluation scale. Plast. Reconstr. Sur 120(7), 1892-1897 (2007).

Lichuan Jiang YYYZ. Analysis aestheti factors in Blepharoplasty Incorporating Chin. J. Med. Aesth. \& Cosmet. 17(6) 477-478 (2011).

Yoo WM, Park SH, Kwag DR. Roo z-epicanthoplasty in asian eyelids. Plasi Reconstr. Surg. 109(6), 2067-2071, 2072 2073 (2002).

Hongwu Zhao FLJS. Anatomic reaso of epicanthus. Chin. J. Med. Aesth. e Cosmet. 3, 176-177 (2001).
Zhang Yuguang YQWX. Anatom construction and mechanics direction o orbicularis oculi effecting superior eyelis morphology. Chin. J. Pract. Aesth. Plasi Surg. 2, 70-72 (2004).

Park JI, Park MS. Park Z Epicanthoplasty. Facial Plast. Surg. Clin North. Am. 15(3), 343-352 (2007).

Mustarde JC. Epicanthus an telecanthus. Br. J. Plast Surg.16, 346-351 (1963).

Adamthwaite J, Sabbagh X Modification of the postauricula fascial flap in Mustarde and Furnas typ otoplasty. Plast. Reconstr. Surg. 126(5) 1786-1787 (2010).

Yoon K. Modification of Mustard technique for correction of epicanthus is Asian patients. Plast. Reconstr. Surg. 97(1) 245 (1996).

Patel BC, Langer P, Anderson RI Modification of Mustarde technique fo correction of epicanthus in Asian patients Plast. Reconstr. Surg. 95(3), 615-61' (1995).

Lin SD. Correction of the epicantha folds using the VM-plasty. Br. J. Plast. Surg. 53(2), 95-99 (2000).
Liu L, Li S, Fan J, et al. Inverted 'VY' advancement medial epicanthoplasty. $J$. Plast. Reconstr. Aesthet. Surg. 65(1), 43-47 (2012).

Zhao YQ, Luo DA. Modified Y-V epicanthoplasty with raised medial canthus in the Asian eyelid. Arch. Facial Plast. Surg. 12(4), 274-276 (2010).

Lee YJ, Baek RM, Song YT, et al. Periciliary Y-V epicanthoplasty. Ann. Plast. Surg. 56(3), 274-278 (2006).

Kao YS, Lin $\mathrm{CH}$, Fang $\mathrm{RH}$. Epicanthoplasty with modified Y-V advancement procedure. Plast. Reconstr. Surg. 102(6), 1835-1841 (1998).

Mulliken JB, Hoopes JE. W-epicanthoplasty. Plast. Reconstr. Surg. 55(4), 435-438 (1975).

Chen W, Li S, Li Y, et al. Medial epicanthoplasty using the palpebral margin incision method. J. Plast. Reconstr. Aesthet. Surg. 62(12), 1621-1626 (2009).

Park J I. Z-epicanthoplasty in Asian eyelids. Plast. Reconstr. Surg. 98(4), 602609 (1996). 